\title{
Nucleic acid sensing in the tumor vasculature
}

Adrian M. Baris ${ }^{1}$, Eugenia Fraile-Bethencourt ${ }^{1}$ and Sudarshan Anand ${ }^{1,2}$

${ }^{1}$ Department of Cell, Developmental \& Cancer Biology,

${ }^{2}$ Department of Radiation Medicine,

Knight Cancer Institute,

2720, S Moody Avenue, Oregon Health \& Science University, Portland, OR 97201

\section{Correspondence to: anands@ohsu.edu}

Simple Summary: Our cells can recognize DNA or RNA from pathogens such as viruses. The proteins that recognize these nucleic acids are known as nucleic acid sensors. Upon activation, they trigger immune responses that result in elimination of the infected cells. Recent research has shown how we can mimic this process in cancer and recruit immune cells against the tumor. Among the different cell types in cancer, endothelial cells play a main role here since the blood vessels are highways for the immune cells. In this review, we discuss two different nucleic acid sensors: the Retinoic acid-Inducible Gene 1 (RIG-I) and the Three prime Repair Exonuclease 1 (TREX1); and how they play a role in endothelial cancer cells. We present some approaches to target these pathways within the cancer blood vessels to disrupt the blood supply and attract immune response to cancers.

Abstract: Endothelial cells form a powerful interface between tissues and immune cells. In fact, one of the underappreciated roles of endothelial cells is to orchestrate immune attention to specific sites. Tumor endothelial cells have a unique ability to dampen the immune responses and thereby maintain an immunosuppressive microenvironment. Recent approaches to trigger immune responses in cancers have focused on activating nucleic acid sensors such as cGAS/STING in combination with immunotherapies. In this review, we present a case for targeting nucleic acid sensing pathways within the tumor vasculature to invigorate tumor immune responses. We introduce two specific nucleic acid sensors, the DNA sensor TREX1 and the RNA sensor RIG-I and discuss their functional roles in the vasculature. Finally, we present perspectives on how these nucleic acid sensors in the tumor endothelium can be targeted in an antiangiogenic and immune activation context. We believe understanding the role of nucleic acid sensing in the tumor vasculature can enhance our ability to design more effective therapies targeting the tumor microenvironment.

Keywords: Nucleic Acid sensors, TREX1, cGAS, STING, RIG-I, Tumor angiogenesis, vascular normalization, vascular inflammation, endothelial cells, tumor microenvironment 


\section{What are Nucleic Acid Sensors?}

In order to protect itself from outside pathogens and other agents, organisms have developed two interlinked forms of defense systems: an innate and an acquired immunity. The innate immune response reacts rapidly to an infection, which can often exponentially multiply long before the adaptive immune response is able to take effect. ${ }^{3}$ These responses should be tightly regulated to prevent dysfunction and damage to the host. The innate immune system responds to Damage Associated Molecular Patterns (DAMPs), which are recognized by Pattern Recognition Receptors (PRRs). Activation of PRRs typically induce a downstream type 1 interferon (IFN-I) and cytokine response. Nucleic acid sensors are a specific type of PRRs that recognize pathogen-derived cytosolic nucleic acids and activate downstream signaling cascades, which produces a proinflammatory response. This response is critical to the ability of nucleic acid sensors stopping pathogens in their tracks. ${ }^{4}$ Nucleic acid sensors are unique in that they recognize and differentiate both exogenous and endogenous nucleic acids. ${ }^{4,5}$ A number of nucleic acid sensors (NAS) play a prominent role in inflammation. ${ }^{6}$ Recent studies have shown that NAS play a large role in endothelial function and dysfunction ${ }^{2}$. Endothelium regulates vascular tone and growth ${ }^{1}$, and is critical in pathologies such as viral infections, cardiovascular disease, and cancer. A better understanding of the role NAS play in endothelial function is critical to improving our knowledge of tumor angiogenesis, as well as cardiovascular and infectious disease. Several outstanding reviews discuss the sensors outlined here (Table 1$)^{7-9}$. In this review, we will focus on the DNA sensor Three prime Repair Exonuclease 1 (TREX1) and RNA sensors in the Retinoic acid-Inducible Gene 1 (RIG-I) like receptors (RLR) pathway (summarized in Figure 1).

\section{What is TREX1?}

DNase III or Trex1, is an exonuclease that degrades exogenous DNA. TREX1 is a member of the DnaQ family of $3^{\prime} \rightarrow 5^{\prime}$ exonucleases. These exonucleases are known for three conserved 
sequence motifs: Exo I, II and III, which are essential for exonuclease function. The TREX1 protein contains a C-terminal domain of about 75 amino acids and a non-repetitive proline-rich region that is not seen in the other TREX protein, TREX2.. ${ }^{10,11}$ TREX1 degrades single strand DNA (ssDNA), double strand DNA (dsDNA) and single strand RNA (ssRNA) in the cytosol. ${ }^{12,13}$ Cytosolic DNA activates the cGAS-STING-TBK1 pathway and leads to the downstream activation of IRF3 and IRF7, which furthermore induce type I interferons. ${ }^{14}$ By degrading cytosolic DNA, TREX1 removes the substrates for cGAS, dampening the nucleic acid sensor response. Therefore, TREX1 is thought to be a negative regulator of interferon signaling and broadly, autoimmune disease. TREX1 interacts with Poly (ADP-ribose) Polymerase (PARP) and is a facilitator of its nuclear translocation and activity ${ }^{10}$ suggesting a putative role in DNA damage responses. Indeed, other studies have shown DNA damage can increase TREX1 expression in a dose dependent manner. ${ }^{16,17}$ In addition to nucleic acid sensing, TREX1 is also involved in the regulation of an ER resident enzyme oligosaccharyltransferase (OST), which contributes to its role in immune regulation and inflammation. ${ }^{18,19}$

\section{What are the biological roles of TREX1?}

DNAse dependent functions

As outlined previously, TREX1 prevents the activation of the cGAS/STING pathway. cGAS is cytosolic cyclic gAMP synthase that is typically in a catalytically inactive autoinhibited state. However, in the presence of DNA, cGAS binds to DNA and undergoes a conformational change which catalyzes the synthesis of cyclic GMP-AMP (cGAMP). cGAMP binds to STING, an endoplasmic-reticulum resident membrane adaptor. This induces a conformational change that activates STING and causes it to traffic to the Golgi, during which TBK1 is activated. The phosphorylation of TBK1 activates its kinase activity, leading to phosphorylation of IRF3 and induces the expression on interferons and inflammatory cytokines. ${ }^{20} \ln 2010$ it was discovered that TREX1 suppresses the interferon response triggered by HIV. In Trex1 deficient mouse cells 
as well as human immune cells with TREX1 RNAi knockdown, HIV infection produced a type 1 IFN response that inhibited HIV replication and spread. ${ }^{21}$ Interestingly, mice deficient in DNAse II, a similar enzyme, die during embryonic development through inflammatory disease. This phenotype is rescued by the loss of STING, as cytosolic DNA was unable to trigger cytokine production through the STING pathway. ${ }^{22}$ These findings suggest that the nuclease activity of TREX1 can be an important negative regulator of cGAS STING pathway.

\section{DNAse independent functions}

TREX1 has a DNAse independent function that suppresses immune activation through regulation of oligosaccharyltransferase (OST) activity. TREX1 is phosphorylated during mitosis, which disrupts its interactions with the OST complex without affecting its DNAse activity. ${ }^{19} 11$

\section{Emerging functions of TREX1}

TREX1 has a wide range of functions in immunity, DNA damage, and cancer. While it has been widely studied, there are still many new functions still being discovered. For example, TREX1 has been shown to inhibit cGAS activation at micronuclei through the degradation of micronuclear DNA. ${ }^{23}$ In addition, cGAS-DNA phase separation sequesters cGAS and prevents access to the negative regulator TREX1. This enhances cGAS DNA sensing activity. ${ }^{24}$

\section{TREX1 knockout mouse}

Trex1 deficient mice exhibit reduced survival and develop inflammatory myocarditis. This often leads to cardiomyopathy and circulatory failure. ${ }^{25}$ These mice also develop lethal interferondriven autoimmune disease, however cGAS deficiency is protective in this instance. ${ }^{26}$ 
Mutations in TREX1 have been linked to several disorders. For example, TREX1 mutations are found in patients with Aicardi-Goutières syndrome (AGS), an encephalopathy that results in severe neurological dysfunction. ${ }^{16}$ Patients with AGS because of a TREX1 mutation typically experience neurological defects such as dystonia, seizures, cortical blindness, and progressive microcephaly, and are more likely to be affected at birth. ${ }^{17}$ There are 5 unique TREX1 mutations that contribute to AGS. These consist of a G314A transition that results in a nonconservative $\mathrm{R}$ to $\mathrm{H}$ substitution predicted to be involved in protein dimerization, a missense T602A mutation in the Exolll motif, and two protein truncating mutations. ${ }^{27}$ Type 1 interferon signaling is shown to be upregulated in most TREX1 AGS patients ${ }^{18}$. TREX1 mutations have also been associated with Familial Chilblain Lupus (FCL), and an upregulation of type 1 interferon genes has been associated in this disease. ${ }^{19}$

\section{How does TREX1 function impact the vasculature?}

Hereditary vascular retinopathy (HVR) is another rare disorder linked to mutations in TREX1 and eventually leads to blindness. It is a microvascular endotheliopathy without any obvious immunological symptoms, however vascular integrity is compromised. ${ }^{20,21}$ TREX1 mutations are also associated with another syndrome Retinal Vasculopathy with Combined Leukodystrophy (RVCL) where capillaries in the retina degenerate leading to vision loss and brain pathology shows DNA damage. ${ }^{28}$ We observed similar functional consequences both in a neonatal ocular angiogenesis model and in the tumor vasculature with miR targeting of TREX1.

Our lab has demonstrated that TREX1 silencing exacerbates DNA damage, cell death, inhibits angiogenesis and induces inflammatory cytokines ${ }^{16}$. Similar to our observations, VanpouilleBox et al showed that TREX1 in tumor cells is induced by high dose radiation and inhibition of TREX1 synergizes with immune checkpoint blockade. ${ }^{29}$ Going beyond TREX1, there is emerging evidence for the function of downstream pathways in the vasculature. STING is expressed in both normal and tumor vasculature. ${ }^{30-32}$ Additionally there is evidence that STING 
signaling in the high endothelial venule can contribute to endothelial-lymphocyte interaction. ${ }^{33}$ Endothelial cell-derived type I IFNs initiate antitumor responses before dendritic cells and CD8 ${ }^{+}$ T cells infiltrate the TME and determine the magnitude of overall immunity. ${ }^{31}$ In humans, mutations in the STING-encoding gene TMEM173, results in a fatal vasculitis, termed STINGassociated vasculopathy with onset in infancy (SAVI). ${ }^{20,34,35}$ Both TBK1 and IRF3 were found to be necessary proangiogenic factors in a high-throughput genomic screen of endothelial cell activity. ${ }^{36}$ Mice deficient in Tbk1 exhibit immune cell infiltrates and an increase in susceptibility to LPS-induced lethality as well as a decrease in IFN- $\beta$ and T-cell expression. ${ }^{37(p 1), 38}$ Irf3 deficient mice experience an altered IFN response as well as the ablation of IFNa and IFN $\beta$ after influenza infection. They also experience an influx of granulocytes in the lung and a decrease in the activation of the adaptive immune response. ${ }^{39}$

What are the RLR family of intracellular RNA sensors? In addition to DNA, PPRs can also recognize RNA in the cytoplasm. The RIG-I like receptor family (RLR) contains three RNA sensors: Retinoic Acid Inducible Gene-I (RIG-I), Melanoma Differentiation-Associated protein 5 (MDA5) and Laboratory of Genetics and Physiology gene 2 (LGP2). These three proteins share a C-terminal domain (CTD) and a helicase domain (HD), but only RIG-I and MDA5 bear the effector domain known as caspase activating and recruiting domain (CARD) in the N-terminal. So that, only RIG-I and MDA5 trigger the IFN-I response through interactions with mitochondrial antiviral signaling proteins (MAVS) ${ }^{40,41,42}$.

\section{What are the substrates (foreign and self)?}

RLRs are activated by cytosolic RNA. RIG-I and MDA5 are similar in structure and function, but they recognize different RNA structures. RIG-I is preferentially activated by blunt-ended 5'ppp short RNAs which bind to the CTD. ${ }^{43,44}$ The CTD of RIG-I has a pocket that specifically binds 
either a 5'-PPP or a 5'-PP. In normal conditions, CARD domain is bound to the HD in a repressing form. Upon RNA recognition, base-paired region of RNA complexes with the HD of RIG-I, releasing the CARD domain. Thus, stable RNA-RIG-I interaction displaces CARDs, which causes multiple RIG-I proteins to oligomerize and become accessible for MAVS signaling. One main player in this process is the E3 ubiquitin ligase TRIM25. TRIM25 ubiquitination is critical to release RIG-I from autorepression. ${ }^{42}$ To interact with MAVS in the mitochondria, the RIG-I complex RIG-I/14-3-3ع/TRIM25 mediates the redistribution or "translocation" of RIG-I from the cytosol to the intracellular membrane compartments. There, RIG-I binds to MAVS through homotypic CARD-CARD interactions. ${ }^{45}$ Once activated, MAVS recruits the tumor necrosis factor receptor associated factors (TRAFs), which are essential to activate interferon regulatory factors 3 and 7 (IRF3, IRF7) and NF-kB mediated response. ${ }^{46}$ Finally, activation of RIG-I results in the expression of cytokines and IFN-I genes, which recruitments the innate immune cells. ${ }^{41,47}$

RLR are able to recognize self-derived RNAs, leading to either enhance or deplete IFN response in a context dependent manner. ${ }^{44} \mathrm{~A}$ recent study showed that mitochondrial DNA double stranded breaks release mitochondrial RNA into the cytoplasm, triggering the RLR dependent immune response. Moreover, following cellular irradiation, mitochondrial DNA breaks synergize with nuclear DNA to promote the immune response. ${ }^{48}$ These emerging studies highlight the potential of RIG-I activation without extrinsic pathogens and could explain the 'sterile inflammation 'in tissues.

\section{What are the biological roles of the RLR family RNA sensors?}

While RIG-I and MDA5 undergo very similar signaling pathways, they do differentially induce type 1 IFN response to different pathogens. ${ }^{40}$ For example, while RIG-I is activated most potently in response to negative-strand viruses such as the influenza virus ${ }^{49}$, MDA5 is activated in response to positive-strand viruses such as hepatitis D virus. ${ }^{50}$ In addition, animal models 
show that RIG-I and MDA5 have functional differences in vivo, as well as distinct molecular immune functions. ${ }^{41}$

\section{Phenotypes in knockout mice}

RIG-I knockout mice show a colitis-like phenotype, reduced Peyer's patches, and show increased effector T cells and decreased naïve T cells. ${ }^{51}$ MAVS and MDA5 KO mice lose type 1 interferon production and suffer early mortality in response to infection with Coxsackie B virus (CVB), which has been associated with myocarditis. ${ }^{52}$ In a study of RLRs in West Nile Virus, RIG-I x MDA5 double knockout mice lacked the innate immune response against the virus infection. Surprisingly, they did not suffer severe pathological damage in tissues during infection, which was similar to animals lacking MAVS. ${ }^{53}$

\section{Associations with human disease}

Singleton-Merten syndrome (SMS) is an autosomal-dominant disorder characterized by aortic calcification, skeletal abnormalities, psoriasis, as well as other conditions. Jang et al performed exome sequencing and found gain-of-function mutations in DDX58, the gene which encodes the RIG-I protein, leads to variable manifestation of SMS, often without the typical dental anomalies. ${ }^{54}$ In addition, gain-of-function mutations of MDA5 have been found in SMS patients with upregulated interferon signature genes. The sustained signaling of MDA5 and RIG-I in SMS patients in possibly due to an increase of protein levels, to the recognition of self-RNA or both. It is believed that excess INF-I and other inflammatory cytokines in the endothelial cells in aortic and mitral valves are critical for the SMS development. ${ }^{55}$

\section{Checks and balances on RIG-I signaling}

Several intricate pathways have evolved to regulate RIG-I signaling in cells. For instance, IncRNAs ATV and Lsm3b have been shown to directly inhibit the RIG-I CTD. ${ }^{56,57}$ Other ncRNAs, 
miR-526a indirectly impact RIG-I by downregulating CYLD, an enzyme that inhibits ubiquitination of RIG-I CARD domain. ${ }^{58}$ There are other miRNAs, such as miR-485, that directly inhibit RIG-I transcripts. ${ }^{59}$

In addition, several post-translational modifications regulate RIG-I function. For example, TRIM38 mediated sumoylation either in the CARD domain or in the CTD can activate RIG-I. ${ }^{60}$ Conversely, acetylation of RIG-I in the CTD at K909 is thought to prevent RIG from binding to viral RNA. Hence, CTD deacetylation by HDACs, especially HDAC6 can enhance RIG-I activation and signaling. ${ }^{61}$

How does RLR function impact the vasculature?

It has been shown that endothelial RIG-I activation leads to endothelial dysfunction. In wild-type mice, endothelial activation of RIG-I leads to endothelial stress, damage, and vessel impairment. After injection with a RIG-I agonist (dsRNA with a triphosphate at the 5 'end), treated mice experienced vascular oxidative stress and increased circulating endothelial microparticle (EMP) numbers, indicating endothelial dysfunction. In addition, after stimulation with the RIG-I agonist, both human coronary endothelial cells (HCAEC) and endothelial progenitor cells (EPC) shows increased reactive oxygen species formation, and HCAES saw increases in proinflammatory cytokines. ${ }^{62}$ Similarly, stimulation of MDA5 led to endothelial apoptosis, formation of reactive oxygen species, and the release of pro-inflammatory cytokines. MDA5 activation in mice endothelium leads to vascular oxidative stress and an increase in circulating endothelial microparticles and endothelial progenitor cells. In addition, chronic MDA5 stimulation exacerbated atherosclerosis. ${ }^{63}$ The activation of Toll-Like Receptor 7 also leads to vascular inflammation and impaired vascular growth. ${ }^{64}$

How can viruses trigger vascular dysfunction through the RIG-I pathway? 
Vascular endothelial cells line the inner surface of blood vessels and provide a barrier between organ systems and blood vessels. This makes them critical during viral infections. Viral infection of endothelial cells gives the virus an opportunity to disperse to other organs, as well as a reservoir for long-term persistence. In addition, viral replication and the immune response in the endothelium leads to an increased tissue permeability as well as inflammation. Altogether ends in vascular and pulmonary disease that further exacerbates the viral disease ${ }^{65}$ Endothelial activation and dysfunction has been shown to serve a necessary mechanistic role in the pathology of severe influenza. ${ }^{66}$ For example, RLRs and TLR7 signaling have been shown to be necessary for cell survival and for restricting virus growth in mice ${ }^{67}$ However, the role of nucleic acid sensors in viral infection needs to be further investigated. RLRs, as well as other aspects of the innate immune system, were found to induce an antiviral innate and adaptive immune response. ${ }^{68}$ Interestingly, in some cases influenza virus has been shown to coopt signaling of TLR7 and RIG-I. ${ }^{69}$

Other viruses rely on endothelial cells for their replication and host response. Particularly relevant of late, endothelial cells have been shown to be essential in the initiation and propagation of SARS-CoV-2, which is responsible of COVID-19 disease. ${ }^{70}$ After the initial phase of infection, some patients experience an overactive inflammatory response which leads to lung damage and increased disease severity. ${ }^{71}$ It has also been proposed that SARS-CoV-2 may cause endothelial dysfunction and pulmonary vascular changes. Thus, endothelial cell injury and dysfunction caused by SARS-CoV-2 may contribute to COVID-19 life-threatening complications. ${ }^{72}$ While there are relevant questions on the role of nucleic acid sensors in the vascular pathologies induced by SARS-CoV-2, they have been well characterized in the vascular inflammatory response to other several infectious pathogens.

How can we target nucleic acid sensors to diminish tumor angiogenesis? 
Tumor angiogenesis involves a number of pro-angiogenic factors in the tumor vasculature. ${ }^{73}$ The tumor vasculature is characterized by disorganized and immature vessels ${ }^{74}$. Tumor cells go into to the vasculature to have access to growth factors as well as spread to other areas of the body. Thus, tumor vasculature must be leaky to allow tumor cells access. ${ }^{75}$ It has been found that tumor angiogenesis is correlated with more metastatic disease in breast cancer. ${ }^{76}$ In this process, ECs were long thought to only provide support for tumors, without playing an active role. However, endothelial cells do regulate a wide variety of cancer cell function. Secretions from quiescent ECs are able to reduce cancer cell proliferation and invasiveness. In fact, altering the secretome of ECs inhibits their ability to suppress cancer progression. ${ }^{77}$ For instance, IFN- $\beta$ is an antiangiogenic cytokine that targets endothelial cells. Endothelial cells are also a major source of type 1 IFNs ${ }^{79-81}$ highlighting a potential feedback inhibitory loop where ECs secrete type I INFs that inhibit angiogenesis.

The notion of anti-angiogenic cancer therapy relies on the idea that removing tumor vasculature will prevent nutrients from entering the tumor, leading to an effective therapy. Conventional antiangiogenic drugs often target agents that promote blood vessel formation and are overexpressed in tumors, such as VEGF-A. However, anti-angiogenesis treatment cannot eradicate the tumor on its own. ${ }^{74}$ The use of chemotherapy or immunotherapy in conjunction with anti-angiogenic treatment can provide a more effective strategy. ${ }^{82}$ Several studies have shown that combining anti-angiogenic therapy with conventional chemotherapy can lead to improved clinical outcomes. ${ }^{83-85}$ Interestingly, STING activation has been shown to normalize the tumor vasculature through increased pericyte coverage and increase in effector T cells across the endothelial barrier, enhancing antitumor immunity. This was most effective when synergized with a VEGF inhibitor, displaying the utility of combining anti-angiogenic drugs with vascular immune therapy. ${ }^{35}$ 
We showed that inhibition of endothelial TREX1 through miR-103 and siRNA was shown to decrease angiogenesis and tumor growth. ${ }^{16}$ Similarly, our recent efforts in the lab have highlighted a role for RIG-I in the tumor vasculature. The use of a 5'PPP containing siVEGF RNA was shown to provide both antiangiogenic therapy and activate RIG-I, leading to an antitumor effect in a murine model of lung cancer. ${ }^{86}$ IRF-1, a known tumor suppressor, has also been shown to inhibit angiogenesis. This has been attributed to a splicing variant involving exon 7 and has implication for anti-angiogenic cancer therapies. ${ }^{87}$ Even recently, Myct1 was identified as a transcription factor in the tumor endothelium and shown to play a dual role in tumor angiogenesis and tumor immunity. ${ }^{88}$

\section{$\underline{\text { Conclusions and future perspectives: }}$}

As proangiogenic molecules have been shown to have immunosuppressive effects, antiangiogenic treatments may also stimulate an immune response. Returning the tumor vasculature to a more normalized state is thought to reverse the immunosuppressive tumor microenvironment and allow the tumor to be treated more effectively. ${ }^{52}$ This has been observed with VEGF inhibitors in tumors, however the success is limited due to immune evasion and development of additional strategies. ${ }^{53,54}$ Anti-angiogenic drugs can be combined with immunotherapies to produce a more potent anti-tumor response, and have been shown to have clinical potential. ${ }^{89}$ We anticipate with more insight into these cytosolic nucleic acid sensing pathways in the tumor vasculature, we will be able to design agents that can both inhibit angiogenesis as well as actively stimulate innate and adaptive immune responses.

\section{Methods:}

Please note that this preprint is a review article and does not report any primary data from 
human or experimental animals.

\section{Acknowledgements}

Work in the Anand lab is supported by funding from NHLBI to S.A. (R01 HL137779 and R01 HL143803). A.B is supported by an institutional T32 GM142619-01 training grant from NIGMS.

\section{Author Contributions}

A.B., E.F-B. and S.A wrote the manuscript. All authors reviewed and edited the manuscript.

\section{Conflicts of Interest}

The authors declare no conflicts of interest.

\section{Figure Legend}

Figure 1: Nucleic Acid Sensors in Endothelial Cells.

RNA sensors RIG-I/MDA5 respond to exogenous RNA and lead to a type I interferon response via the MAVS pathway in ECs. Conversely, TREX1 degrades ssDNA or dsDNA in the cytosol thereby preventing activation of cGAS/STING pathway that activates type I interferons. Activation of RIG-I pathway or inhibition of TREX1 pathway will inhibit tumor angiogenesis by upregulating interferon responses. 


\section{$\underline{\text { References }}$}

1. Lüscher TF, Tanner FC. Endothelial Regulation of Vascular Tone and Growth. Am J Hypertens. 1993;6(7_Pt_2):283S-293S. doi:10.1093/ajh/6.7.283S

2. Bhagwani A, Thompson AAR, Farkas L. When Innate Immunity Meets Angiogenesis-The Role of Toll-Like Receptors in Endothelial Cells and Pulmonary Hypertension. Front Med. 2020;7. doi:10.3389/fmed.2020.00352

3. Alberts B, Johnson A, Lewis J, Raff M, Roberts K, Walter P. Innate Immunity. Mol Biol Cell 4th Ed. Published online 2002. Accessed November 19, 2020. https://www.ncbi.nlm.nih.gov/books/NBK26846/

4. Ori D, Murase M, Kawai T. Cytosolic nucleic acid sensors and innate immune regulation. Int Rev Immunol. 2017;36(2):74-88. doi:10.1080/08830185.2017.1298749

5. Takeuchi O, Akira S. Pattern Recognition Receptors and Inflammation. Cell. 2010;140(6):805-820. doi:10.1016/j.cell.2010.01.022

6. Khan S, Godfrey V, Zaki MdH. Chapter Seven - Cytosolic Nucleic Acid Sensors in Inflammatory and Autoimmune Disorders. In: Vanpouille-Box C, Galluzzi L, eds. International Review of Cell and Molecular Biology. Vol 344. Nucleic Acid Sensing and Immunity, Part A. Academic Press; 2019:215-253. doi:10.1016/bs.ircmb.2018.10.002

7. Vanpouille-Box C, Hoffmann JA, Galluzzi L. Pharmacological modulation of nucleic acid sensors - therapeutic potential and persisting obstacles. Nat Rev Drug Discov. 2019;18(11):845-867. doi:10.1038/s41573-019-0043-2

8. Tiered DNA sensors for escalating responses | Science. Accessed June 7, 2021. https://science.sciencemag.org/content/365/6460/1375.full

9. Jiao $\mathrm{H}$, Wachsmuth $\mathrm{L}$, Kumari $\mathrm{S}$, et al. Z-nucleic-acid sensing triggers ZBP1-dependent necroptosis and inflammation. Nature. 2020;580(7803):391-395. doi:10.1038/s41586-0202129-8

10. Höss M, Robins P, Naven TJP, Pappin DJC, Sgouros J, Lindahl T. A human DNA editing enzyme homologous to the Escherichia coli DnaQ/MutD protein. EMBO J. 1999;18(13):3868-3875. doi:10.1093/emboj/18.13.3868

11. de Silva U, Choudhury S, Bailey SL, Harvey S, Perrino FW, Hollis T. The Crystal Structure of TREX1 Explains the 3' Nucleotide Specificity and Reveals a Polyproline II Helix for Protein Partnering*. J Biol Chem. 2007;282(14):10537-10543.

doi:10.1074/jbc.M700039200

12. Grieves JL, Fye JM, Harvey S, Grayson JM, Hollis T, Perrino FW. Exonuclease TREX1 degrades double-stranded DNA to prevent spontaneous lupus-like inflammatory disease. Proc Natl Acad Sci. 2015;112(16):5117-5122. doi:10.1073/pnas.1423804112

13. Yuan F, Dutta $T$, Wang $L$, et al. Human DNA Exonuclease TREX1 Is Also an Exoribonuclease That Acts on Single-stranded RNA. J Biol Chem. 2015;290(21):1334413353. doi:10.1074/jbc.M115.653915 
14. Stetson DB, Ko JS, Heidmann T, Medzhitov R. Trex1 Prevents Cell-Intrinsic Initiation of Autoimmunity. Cell. 2008;134(4):587-598. doi:10.1016/j.cell.2008.06.032

15. Miyazaki T, Kim Y-S, Yoon J, Wang H, Suzuki T, Morse HC. The 3'-5' DNA exonuclease TREX1 directly interacts with poly(ADP-ribose) polymerase-1 (PARP1) during the DNA damage response. J Biol Chem. 2014;289(47):32548-32558. doi:10.1074/jbc.M114.547331

16. Wilson R, Espinosa-Diez C, Kanner N, et al. MicroRNA regulation of endothelial TREX1 reprograms the tumour microenvironment. Nat Commun. 2016;7(1):1-10. doi:10.1038/ncomms 13597

17. Vanpouille-Box C, Formenti SC, Demaria S. TREX1 dictates the immune fate of irradiated cancer cells. Oncoimmunology. 2017;6(9). doi:10.1080/2162402X.2017.1339857

18. Hasan M, Fermaintt CS, Gao N, et al. Cytosolic nuclease TREX1 regulates oligosaccharyltransferase activity independent of nuclease activity to suppress immune activation. Immunity. 2015;43(3):463-474. doi:10.1016/j.immuni.2015.07.022

19. Kucej M, Fermaintt CS, Yang K, Irizarry-Caro RA, Yan N. Mitotic Phosphorylation of TREX1 C Terminus Disrupts TREX1 Regulation of the Oligosaccharyltransferase Complex. Cell Rep. 2017;18(11):2600-2607. doi:10.1016/j.celrep.2017.02.051

20. Chen $Q$, Sun L, Chen ZJ. Regulation and function of the cGAS-STING pathway of cytosolic DNA sensing. Nat Immunol. 2016;17(10):1142-1149. doi:10.1038/ni.3558

21. Yan N, Regalado-Magdos AD, Stiggelbout B, Lee-Kirsch MA, Lieberman J. The cytosolic exonuclease TREX1 inhibits the innate immune response to human immunodeficiency virus type 1. Nat Immunol. 2010;11(11):1005-1013. doi:10.1038/ni.1941

22. Ahn J, Gutman D, Saijo S, Barber GN. STING manifests self DNA-dependent inflammatory disease. Proc Natl Acad Sci. 2012;109(47):19386-19391. doi:10.1073/pnas.1215006109

23. Mohr L, Toufektchan E, von Morgen P, Chu K, Kapoor A, Maciejowski J. ER-directed TREX1 limits cGAS activation at micronuclei. Mol Cell. 2021;81(4):724-738.e9. doi:10.1016/j.molcel.2020.12.037

24. Zhou W, Mohr L, Maciejowski J, Kranzusch PJ. cGAS phase separation inhibits TREX1mediated DNA degradation and enhances cytosolic DNA sensing. Mol Cell. 2021;81(4):739-755.e7. doi:10.1016/j.molcel.2021.01.024

25. Morita M, Stamp G, Robins P, et al. Gene-Targeted Mice Lacking the Trex1 (DNase III) 3' $\rightarrow$ 5' DNA Exonuclease Develop Inflammatory Myocarditis. Mol Cell Biol. 2004;24(15):6719-6727. doi:10.1128/MCB.24.15.6719-6727.2004

26. Gray EE, Treuting PM, Woodward JJ, Stetson DB. Cutting Edge: cGAS Is Required for Lethal Autoimmune Disease in the Trex1-Deficient Mouse Model of Aicardi-Goutières Syndrome. J Immunol. 2015;195(5):1939-1943. doi:10.4049/jimmunol.1500969 
27. Crow YJ, Hayward BE, Parmar R, et al. Mutations in the gene encoding the 3'-5' DNA exonuclease TREX1 cause Aicardi-Goutières syndrome at the AGS1 locus. Nat Genet. 2006;38(8):917-920. doi:10.1038/ng1845

28. Richards $A$, van den Maagdenberg AMJM, Jen JC, et al. C-terminal truncations in human 3'-5' DNA exonuclease TREX1 cause autosomal dominant retinal vasculopathy with cerebral leukodystrophy. Nat Genet. 2007;39(9):1068-1070. doi:10.1038/ng2082

29. DNA exonuclease Trex1 regulates radiotherapy-induced tumour immunogenicity | Nature Communications. Accessed April 12, 2021. https://www.nature.com/articles/ncomms15618

30. Corrales L, Glickman LH, McWhirter SM, et al. Direct Activation of STING in the Tumor Microenvironment Leads to Potent and Systemic Tumor Regression and Immunity. Cell Rep. 2015;11(7):1018-1030. doi:10.1016/j.celrep.2015.04.031

31. STING activation of tumor endothelial cells initiates spontaneous and therapeutic antitumor immunity | PNAS. Accessed July 8, 2020.

https://www.pnas.org/content/112/50/15408.short

32. STING is an endoplasmic reticulum adaptor that facilitates innate immune signalling | Nature. Accessed May 12, 2021. https://www.nature.com/articles/nature07317

33. STING expression and response to treatment with STING ligands in premalignant and malignant disease. Accessed May 12, 2021.

https://journals.plos.org/plosone/article?id=10.1371/journal.pone.0187532

34. Activated STING in a Vascular and Pulmonary Syndrome | NEJM. Accessed May 12, 2021. https://www.nejm.org/doi/full/10.1056/nejmoa1312625

35. JCI - STING activation reprograms tumor vasculatures and synergizes with VEGFR2 blockade. Accessed April 13, 2021. https://www.jci.org/articles/view/125413

36. Korherr $\mathrm{C}$, Gille H, Schäfer R, et al. Identification of proangiogenic genes and pathways by high-throughput functional genomics: TBK1 and the IRF3 pathway. Proc Natl Acad Sci. 2006;103(11):4240-4245. doi:10.1073/pnas.0511319103

37. Yu T, Yi Y-S, Yang Y, Oh J, Jeong D, Cho JY. The Pivotal Role of TBK1 in Inflammatory Responses Mediated by Macrophages. Mediators Inflamm. 2012;2012:e979105. doi:10.1155/2012/979105

38. Mice lacking Tbk1 activity exhibit immune cell infiltrates in multiple tissues and increased susceptibility to LPS-induced lethality - Marchlik - 2010 - Journal of Leukocyte Biology Wiley Online Library. Accessed May 12, 2021. https://jlb.onlinelibrary.wiley.com/doi/full/10.1189/jlb.0210071

39. Hatesuer B, Hoang HTT, Riese P, et al. Deletion of Irf3 and Irf7 Genes in Mice Results in Altered Interferon Pathway Activation and Granulocyte-Dominated Inflammatory Responses to Influenza A Infection. J Innate Immun. 2017;9(2):145-161. doi:10.1159/000450705 
40. Kell AM, Gale M. RIG-I in RNA virus recognition. Virology. 2015;479:110-121. doi:10.1016/j.virol.2015.02.017

41. Brisse M, Ly H. Comparative Structure and Function Analysis of the RIG-I-Like Receptors: RIG-I and MDA5. Front Immunol. 2019;10. doi:10.3389/fimmu.2019.01586

42. Wu B, Hur S. How RIG-I like receptors activate MAVS. Curr Opin Virol. 2015;12:91-98. doi:10.1016/j.coviro.2015.04.004

43. Kolakofsky D, Kowalinski E, Cusack S. A structure-based model of RIG-I activation. RNA. 2012;18(12):2118-2127. doi:10.1261/rna.035949.112

44. Stok JE, Quiroz MEV, Veen AG van der. Self RNA Sensing by RIG-I-like Receptors in Viral Infection and Sterile Inflammation. J Immunol. 2020;205(4):883-891. doi:10.4049/jimmunol.2000488

45. Rehwinkel J, Gack MU. RIG-I-like receptors: their regulation and roles in RNA sensing. Nat Rev Immunol. 2020;20(9):537-551. doi:10.1038/s41577-020-0288-3

46. Fitzgerald KA, McWhirter SM, Faia KL, et al. IKKE and TBK1 are essential components of the IRF3 signaling pathway. Nat Immunol. 2003;4(5):491-496. doi:10.1038/ni921

47. $S P, Q S, P N$, et al. Induction of IRF-3 and IRF-7 phosphorylation following activation of the RIG-I pathway. Cell Mol Biol Noisy--Gd Fr. 2006;52(1):17-28.

48. Tigano M, Vargas DC, Tremblay-Belzile S, Fu Y, Sfeir A. Nuclear sensing of breaks in mitochondrial DNA enhances immune surveillance. Nature. 2021;591(7850):477-481. doi:10.1038/s41586-021-03269-w

49. Weber-Gerlach M, Weber F. Standing on three legs: antiviral activities of RIG-I against influenza viruses. Curr Opin Immunol. 2016;42:71-75. doi:10.1016/j.coi.2016.05.016

50. Hepatitis $D$ virus replication is sensed by MDA5 and induces IFN- $\beta / \lambda$ responses in hepatocytes - Journal of Hepatology. Accessed July 8, 2020. https://www.journal-ofhepatology.eu/article/S0168-8278(18)30143-0/fulltext

51. Wang $\mathrm{Y}$, Zhang $\mathrm{H}-\mathrm{X}$, Sun $\mathrm{Y}-\mathrm{P}$, et al. Rig-I -/- mice develop colitis associated with downregulation of Gai2. Cell Res. 2007;17(10):858-868. doi:10.1038/cr.2007.81

52. Wang JP, Cerny A, Asher DR, Kurt-Jones EA, Bronson RT, Finberg RW. MDA5 and MAVS Mediate Type I Interferon Responses to Coxsackie B Virus. J Virol. 2010;84(1):254260. doi:10.1128/JVI.00631-09

53. Errett JS, Suthar MS, McMillan A, Diamond MS, Gale M. The essential, nonredundant roles of RIG-I and MDA5 in detecting and controlling West Nile virus infection. $J$ Virol. 2013;87(21):11416-11425. doi:10.1128/JVI.01488-13

54. Jang M-A, Kim EK, Now H, et al. Mutations in DDX58, which Encodes RIG-I, Cause Atypical Singleton-Merten Syndrome. Am J Hum Genet. 2015;96(2):266-274. doi:10.1016/j.ajhg.2014.11.019 
55. Lu C, MacDougall M. RIG-I-Like Receptor Signaling in Singleton-Merten Syndrome. Front Genet. 2017;8. doi:10.3389/fgene.2017.00118

56. Jiang M, Zhang S, Yang Z, et al. Self-Recognition of an Inducible Host IncRNA by RIG-I Feedback Restricts Innate Immune Response. Cell. 2018;173(4):906-919.e13. doi:10.1016/j.cell.2018.03.064

57. Ma H, Han P, Ye W, et al. The Long Noncoding RNA NEAT1 Exerts Antihantaviral Effects by Acting as Positive Feedback for RIG-I Signaling. J Virol. 2017;91(9). doi:10.1128/JVI.02250-16

58. Xu C, He X, Zheng Z, et al. Downregulation of MicroRNA miR-526a by Enterovirus Inhibits RIG-I-Dependent Innate Immune Response. J Virol. 2014;88(19):11356-11368. doi:10.1128/JVI.01400-14

59. The microRNA miR-485 targets host and influenza virus transcripts to regulate antiviral immunity and restrict viral replication | Science Signaling. Accessed May 26, 2021. https://stke.sciencemag.org/content/8/406/ra126.long

60. Hu M-M, Liao C-Y, Yang Q, Xie X-Q, Shu H-B. Innate immunity to RNA virus is regulated by temporal and reversible sumoylation of RIG-I and MDA5. J Exp Med. 2017;214(4):973989. doi:10.1084/jem.20161015

61. Liu HM, Jiang F, Loo YM, et al. Regulation of Retinoic Acid Inducible Gene-I (RIG-I) Activation by the Histone Deacetylase 6. EBioMedicine. 2016;9:195-206. doi:10.1016/j.ebiom.2016.06.015

62. Asdonk T, Motz I, Werner N, et al. Endothelial RIG-I activation impairs endothelial function. Biochem Biophys Res Commun. 2012;420(1):66-71. doi:10.1016/j.bbrc.2012.02.116

63. Asdonk T, Steinmetz M, Krogmann A, et al. MDA-5 activation by cytoplasmic doublestranded RNA impairs endothelial function and aggravates atherosclerosis. J Cell Mol Med. 2016;20(9):1696-1705. doi:10.1111/jcmm.12864

64. O Krogmann A, Lüsebrink E, Lahrmann C, Flender A, Nickenig G, Zimmer S. Toll-Like Receptor 7 Stimulation Promotes the Development of Atherosclerosis in Apolipoprotein EDeficient Mice. Int Heart J. 2020;61(2):364-372. doi:10.1536/ihj.19-365

65. Goodrum F, Bughio F. Viral infection at the endothelium. Oncotarget. 2015;6(29):2654126542.

66. Armstrong SM, Darwish I, Lee WL. Endothelial activation and dysfunction in the pathogenesis of influenza A virus infection. Virulence. 2013;4(6):537-542. doi:10.4161/viru.25779

67. Iwasaki A, Pillai PS. Innate immunity to influenza virus infection. Nat Rev Immunol. 2014;14(5):315-328. doi:10.1038/nri3665

68. Koyama S, Ishii KJ, Kumar H, et al. Differential role of TLR- and RLR-signaling in the immune responses to influenza A virus infection and vaccination. $\mathrm{J}$ Immunol Baltim Md 1950. 2007;179(7):4711-4720. doi:10.4049/jimmunol.179.7.4711 
69. Pang IK, Pillai PS, Iwasaki A. Efficient influenza A virus replication in the respiratory tract requires signals from TLR7 and RIG-I. Proc Natl Acad Sci. 2013;110(34):13910-13915. doi:10.1073/pnas. 1303275110

70. Teuwen L-A, Geldhof V, Pasut A, Carmeliet P. COVID-19: the vasculature unleashed. Nat Rev Immunol. 2020;20(7):389-391. doi:10.1038/s41577-020-0343-0

71. Wu Z, McGoogan JM. Characteristics of and Important Lessons From the Coronavirus Disease 2019 (COVID-19) Outbreak in China: Summary of a Report of 72314 Cases From the Chinese Center for Disease Control and Prevention. JAMA. 2020;323(13):1239-1242. doi:10.1001/jama.2020.2648

72. Huertas A, Montani D, Savale L, et al. Endothelial cell dysfunction: a major player in SARS-CoV-2 infection (COVID-19)? Eur Respir J. 2020;56(1).

doi:10.1183/13993003.01634-2020

73. Kabiraj A, Jaiswal R, Singh A, Gupta J, Singh A, Samadi FM. Immunohistochemical evaluation of tumor angiogenesis and the role of mast cells in oral squamous cell carcinoma. J Cancer Res Ther. 2018;14(3):495-502. doi:10.4103/0973-1482.163693

74. Stockmann C, Schadendorf D, Klose R, Helfrich I. The Impact of the Immune System on Tumor: Angiogenesis and Vascular Remodeling. Front Oncol. 2014;4. doi:10.3389/fonc.2014.00069

75. Fidler IJ. Tumor Heterogeneity and the Biology of Cancer Invasion and Metastasis. Cancer Res. 1978;38(9):2651-2660.

76. Weidner N, Semple JP, Welch WR, Folkman J. Tumor Angiogenesis and Metastasis Correlation in Invasive Breast Carcinoma.

http://dx.doi.org/10.1056/NEJM199101033240101. doi:10.1056/NEJM199101033240101

77. Franses JW, Baker AB, Chitalia VC, Edelman ER. Stromal Endothelial Cells Directly Influence Cancer Progression. Sci Transl Med. 2011;3(66):66ra5-66ra5.

doi:10.1126/scitranslmed.3001542

78. Poeck H, Besch R, Maihoefer C, et al. 5'-triphosphate-siRNA: turning gene silencing and Rig-I activation against melanoma. Nat Med. 2008;14(11):1256-1263.

doi:10.1038/nm.1887

79. Continuous Delivery of IFN- $\beta$ Promotes Sustained Maturation of Intratumoral Vasculature | Molecular Cancer Research. Accessed May 3, 2021.

https://mcr.aacrjournals.org/content/5/6/531.short

80. Jia H, Thelwell C, Dilger P, Bird C, Daniels S, Wadhwa M. Endothelial cell functions impaired by interferon in vitro: Insights into the molecular mechanism of thrombotic microangiopathy associated with interferon therapy. Thromb Res. 2018;163:105-116. doi:10.1016/j.thromres.2018.01.039

81. Inhibition of Angiogenesis by Interferons: Effects on Tumor-and Lymphocyte-induced Vascular Responses | Cancer Research. Accessed May 3, 2021. https://cancerres.aacrjournals.org/content/47/19/5155.short 
82. Rajabi M, Mousa SA. The Role of Angiogenesis in Cancer Treatment. Biomedicines. 2017;5(2). doi:10.3390/biomedicines5020034

83. Quantifying the effects of antiangiogenic and chemotherapy drug combinations on drug delivery and treatment efficacy. Accessed February 10, 2021.

https://journals.plos.org/ploscompbiol/article?id=10.1371/journal.pcbi.1005724

84. Letellier C, Sasmal SK, Draghi C, Denis F, Ghosh D. A chemotherapy combined with an anti-angiogenic drug applied to a cancer model including angiogenesis. Chaos Solitons Fractals. 2017;99:297-311. doi:10.1016/j.chaos.2017.04.013

85. Robert NJ, Diéras V, Glaspy J, et al. RIBBON-1: randomized, double-blind, placebocontrolled, phase III trial of chemotherapy with or without bevacizumab for first-line treatment of human epidermal growth factor receptor 2-negative, locally recurrent or metastatic breast cancer. J Clin Oncol Off J Am Soc Clin Oncol. 2011;29(10):1252-1260. doi:10.1200/JCO.2010.28.0982

86. Yuan D, Xia M, Meng G, Xu C, Song Y, Wei J. Anti-angiogenic efficacy of 5'-triphosphate siRNA combining VEGF silencing and RIG-I activation in NSCLCs. Oncotarget. 2015;6(30):29664-29674. doi:10.18632/oncotarget.4869

87. Lee JH, Chun T, Park S-Y, Rho SB. Interferon regulatory factor-1 (IRF-1) regulates VEGFinduced angiogenesis in HUVECs. Biochim Biophys Acta BBA - Mol Cell Res. 2008;1783(9):1654-1662. doi:10.1016/j.bbamcr.2008.04.006

88. Kabir AU, Subramanian M, Lee DH, et al. Dual role of endothelial Myct1 in tumor angiogenesis and tumor immunity. Sci Transl Med. 2021;13(583).

doi:10.1126/scitranslmed.abb6731

89. Khan KA, Kerbel RS. Improving immunotherapy outcomes with anti-angiogenic treatments and vice versa. Nat Rev Clin Oncol. 2018;15(5):310-324. doi:10.1038/nrclinonc.2018.9 
Baris et al, Figure 1 


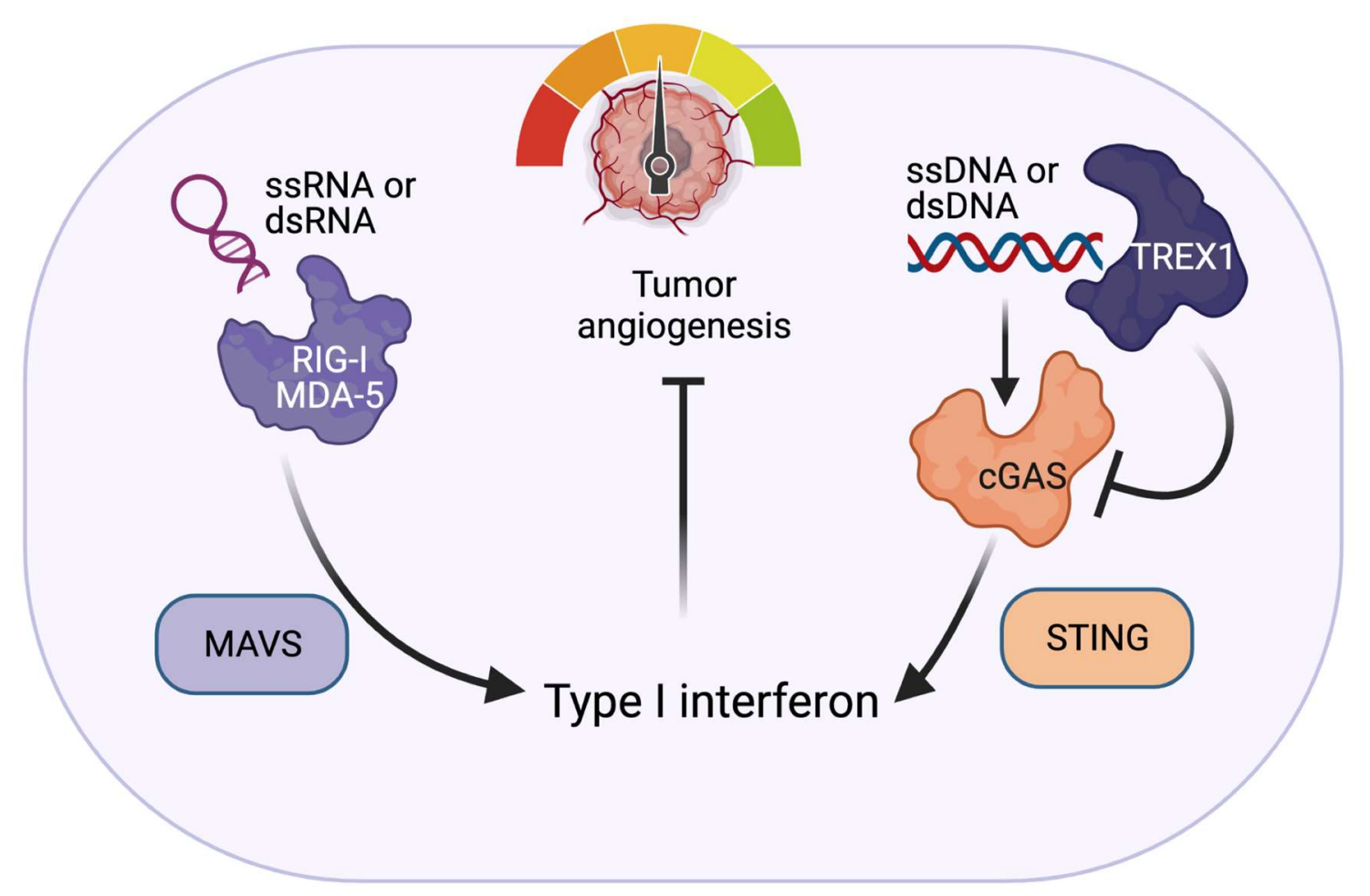

Baris et al, Table 1 


\begin{tabular}{|lllll|}
\hline Gene & Protein & \multicolumn{1}{c}{ Substrate } & \multicolumn{1}{c|}{ Function } & \multicolumn{1}{c|}{ Reference } \\
\hline Cgas & cGAS & dsDNA & Apoptosis & Schlee et al, 2016 \\
TLR9 & TLR9 & RNA-DNA hybrid & Inflammation & Schlee et al, 2016 \\
TREX1 & TREX1 & dsDNA & Immune suppression & Vanpouille-Box et al, 2019 \\
AIM2 & AIM2 & dsDNA & Pyroptosis & Schlee et al, 2016 \\
IFI16 & IFI16 & dsDNA & Pyroptosis & Vanpouille-Box et al, 2019 \\
IFIH1 & MDA5 & dsRNA & Apoptosis & Vanpouille-Box et al, 2019 \\
DDX58 & RIG-I & dsRNA & Inflammation & Vanpouille-Box et al, 2019 \\
TLR3 & TLR3 & dsRNA & Nectroptosis & Vanpouille-Box et al, 2019 \\
ZBP1 & ZBP1 & B-DNA, Z-DNA & Necroptosis & Jiao et al, 2020 \\
\hline
\end{tabular}

